\title{
Frequency and molecular characteristics of ciprofloxacin- and rifampicin-resistant Helicobacter pylori from gastric infections in the UK
}

\author{
Stephanie A. Chisholm and Robert J. Owen \\ Gastrointestinal, Emerging and Zoonotic Infections Department, Centre for Infections, Health \\ Protection Agency, 61 Colindale Avenue, London NW9 5HT, UK
}

Correspondence

Robert J. Owen

robert.owen@hpa.org.uk

Received 20 March 2009

Accepted 6 July 2009

\section{INTRODUCTION}

Helicobacter pylori infection can lead to the development of peptic ulcer disease, lymphoproliferative disorders and early-stage gastric carcinoma (Go, 2002; Suerbaum \& Michetti, 2002). Current guidelines recommend a triple therapy comprising a proton pump inhibitor and clarithromycin and either metronidazole or amoxicillin as a first-line regimen, and a quadruple therapy comprising a proton pump inhibitor plus bismuth, metronidazole and tetracycline as a second-line strategy (Malfertheiner et al., 2007). Antibiotic resistance, particularly to clarithromycin and metronidazole, is a major contributing factor in treatment failure (Dore et al., 2000; Jenks, 2002), and infection can persist in as many as $20 \%$ of patients following treatment (Gisbert \& Pajares, 2005). Such limitations have led to the development of alternative 'rescue' therapies using agents not used in first- or secondline therapies, such as the fluoroquinolones moxifloxacin and levofloxacin, and rifabutin, a rifamycin derivative commonly used in the treatment of mycobacterial infections but with high efficacy against $H$. pylori (Gisbert \& Pajares, 2005; Toracchio et al., 2005).

Regimes containing these alternative antimicrobial agents have proved successful for the management of persistent $H$. pylori infections. Studies in Europe, the USA, Asia and several other countries have monitored fluoroquinolone resistance rates in different patient groups including

Abbreviation: QRDR, quinolone resistance-determining region. children and have reported rates of up to $33 \%$ (Cabrita et al., 2000; Tankovic et al., 2003; Falsafi et al., 2004; Fujimura et al., 2004; Kim et al., 2004; Sherif et al., 2004; Bogaerts et al., 2006; Kumala \& Rani, 2006; Miyachi et al., 2006; Carothers et al., 2007; Cattoir et al., 2007; Glocker et al., 2007b; Zullo et al., 2007; Boyanova et al., 2008; Lee et al., 2008). In contrast, information on rifampicin/ rifabutin resistance in $H$. pylori is more limited, as no examples of resistance were found in studies of German (Heep et al., 1999), Japanese (Fujimura et al., 2002) or Italian (Toracchio et al., 2005) isolates, although a subsequent German study reported a resistance rate of $1.4 \%$ (Glocker et al., 2007a).

There is currently no information on either fluoroquinolone or rifamycin resistance of $H$. pylori from patients in the UK, so the aim of our study was to report on the frequencies of resistance to ciprofloxacin and rifampicin using isolates from adult patients, including those with refractive $H$. pylori infection. We also detailed the gene mutations associated with such resistances because of their importance in providing the basis of future rapid molecular diagnostic tests - an approach already successfully applied to clarithromycin for which the molecular basis of resistance in $H$. pylori is well understood (Chisholm \& Owen, 2008). Ciprofloxacin inhibits subunit A of DNA gyrase (Reece \& Maxwell, 1991), and in $H$. pylori, resistance is reported to arise from mutations in the 'quinolone resistance-determining region' (QRDR) of gyrA (Moore et al., 1995) with a number of mutations associated 
with higher resistance levels (Bogaerts et al., 2006; Miyachi et al., 2006). Rifampicin, by contrast, exerts its antibacterial effect by binding to the $\beta$-subunit of the DNA-directed RNA polymerase encoded by the RNA polymerase subunit $\mathrm{B}$ gene $(r p o B)$ gene, with resistance in $H$. pylori developing as a result of specific mutations in $r p o B$ (Heep et al., 1999; Wang et al., 2001). Here, we present information on the presence of mutations associated with antibiotic-resistant phenotypes.

\section{METHODS}

Bacterial isolates. $H$. pylori isolates cultured over a 6-year period (2001-2006) from gastric biopsies of 255 adult dyspeptic patients were included in this study. The majority of isolates $(171 / 255,67 \%)$ were cultured from gastric biopsies referred to our laboratory for routine testing by previously described methods (Chisholm et al., 2007). The biopsies were referred from 76 different hospitals located across England, Scotland and Wales, and were from patients undergoing gastroendoscopy as part of a local routine clinical investigation. Available information indicated that $70 \%(n=121)$ of these patients had at least one failed eradication therapy attempt. The treatment histories of the remaining 50 patients with culture-positive biopsies were unknown. An additional 84 isolates (33\%) in the study were received as cultures after primary isolation had been performed in local microbiology laboratories, as described previously (Elviss et al., 2004, 2005). Available information indicated that these isolates were from gastric biopsies from patients attending endoscopy clinics for routine investigation prior to specific anti-H. pylori eradication therapy. The history of any previous treatment regimens used was unknown. All 255 isolates were preserved at $-80{ }^{\circ} \mathrm{C}$.

Antibiotic susceptibility testing. The isolates had been tested previously for susceptibility to metronidazole, clarithromycin, tetracycline and amoxicillin using established methods used longitudinally in our accredited laboratory (Chisholm et al., 2007). For the present study, susceptibilities to ciprofloxacin and rifampicin were tested by Etest and/or disc diffusion methods. Briefly, exponential (48 h) bacterial growth was suspended in maximum recovery diluent (Oxoid) to a concentration of $\sim 10^{8}$ c.f.u. $\mathrm{ml}^{-1}$ (MacFarland standard 4) and inoculated onto pre-dried Columbia agar base plates containing defibrinated horse blood $(10 \%, \mathrm{v} / \mathrm{v})$. Ciprofloxacin and rifampicin Etests ( $\mathrm{AB}$ Biodisk) and/or antibiotic discs (Oxoid) containing $1 \mu \mathrm{g}$ ciprofloxacin or $5 \mu \mathrm{g}$ rifampicin were applied to the surface of these plates and any inhibition of bacterial growth was recorded following $48 \mathrm{~h}$ microaerophilic incubation $\left(4 \% \mathrm{O}_{2}, 5 \%\right.$ $\mathrm{CO}_{2}, 86 \% \mathrm{~N}_{2}, 5 \% \mathrm{H}_{2}$ ) at $37{ }^{\circ} \mathrm{C}$ in a MACS VA500 Microaerophilic Workstation (Don Whitley Scientific).

Initially, MICs for ciprofloxacin and rifampicin for 115 isolates were determined by Etest. Isolates were classified as ciprofloxacin resistant $\left(\geqslant 1 \mathrm{mg} \mathrm{l}^{-1}\right)$ or sensitive $\left(<1 \mathrm{mg} \mathrm{l}^{-1}\right)$ and rifampicin resistant $\left(\geqslant 4 \mathrm{mg} \mathrm{l}^{-1}\right)$ or sensitive $\left(<4 \mathrm{mg} \mathrm{l}^{-1}\right)$ based on criteria indicated by previous investigators (Cabrita et al., 2000; Heep et al., 2000a; Tankovic et al., 2003). Thirty-three of these 115 isolates were tested additionally by the disc diffusion method to establish appropriate zone size cut-offs to define resistant and sensitive isolates. A further 139 isolates received in 2006 were screened prospectively by the disc diffusion method and MICs were then determined by Etest for any isolates resistant by disc diffusion.

Sequencing of resistance-associated gene regions. Genomic DNA was extracted from exponential $(48 \mathrm{~h})$ cultures using a MagNA Pure System LC Total NA Isolation kit (Roche Diagnostics), according to the manufacturer's instructions. To test for markers of ciprofloxacin resistance, a $246 \mathrm{bp}$ fragment of the gyrA gene containing the QRDR was amplified by PCR as described previously (Fujimura et al., 2004). To test for markers of rifampicin resistance, two fragments of $r p o B$ were amplified using previously described primers: the first fragment contained the amino acid V149 region (Heep et al., 2000b), and the second fragment contained cluster regions I and II (Heep et al., 1999). All amplicons were purified by using a Whatman 96-Well PCR Cleanup kit (VWR International), according to the manufacturer's protocol, and quantified by gel electrophoresis. The purified fragments were sequenced using a CEQ800 Genetic Analysis System (Beckman Coulter). Sequences were trimmed and analysed using Bionumerics version 2.0 and GeneBase version 1.0 software (Applied Maths).

\section{RESULTS AND DISCUSSION}

\section{Characterization of the susceptibility of the study population to ciprofloxacin}

The MICs for ciprofloxacin of 115 isolates of $H$. pylori had a bimodal distribution (Fig. 1a), with a clear separation between sensitive (MICs ranging from 0.016 to $0.5 \mathrm{mg} \mathrm{l}^{-1}$ ) and resistant (MICs ranging from 4 to $32 \mathrm{mg} \mathrm{l}^{-1}$ ) isolates at the $1 \mathrm{mg} \mathrm{l}^{-1}$ breakpoint. The MIC 90 and MIC $_{50}$ values of the isolates examined were 0.38 and $0.094 \mathrm{mg} \mathrm{l}^{-1}$, respectively, with median and modal MICs of $0.094 \mathrm{mg}$ $1^{-1}$, indicating a predominantly susceptible population. Zones of inhibition observed by disc diffusion testing ranged from 20 to $54 \mathrm{~mm}$ (mean $41 \mathrm{~mm}$, mode $42 \mathrm{~mm}$ ) for ciprofloxacin-sensitive isolates $(n=24)$ and from 0 to $19 \mathrm{~mm}$ (mean $6 \mathrm{~mm}$, mode $0 \mathrm{~mm}$ ) for resistant isolates $(n=10)$. With one exception, all zone sizes of $\leqslant 14 \mathrm{~mm}$ were associated with MICs of $12 \mathrm{mg} \mathrm{l}^{-1}$ or more, whilst larger zones of $19 \mathrm{~mm}$ were associated with lower MICs (2 and $4 \mathrm{mg} \mathrm{l}^{-1}$ ) (Table 1).

To date, most studies have monitored fluoroquinolone resistance in $H$. pylori by Etest or to a lesser extent by the agar dilution method; the single study that used disc diffusion did not state the cut-off for resistance (Falsafi et al., 2004). The data we obtained for $1 \mu \mathrm{g}$ ciprofloxacin discs demonstrated that zones of inhibition of $\leqslant 14 \mathrm{~mm}$ by disc diffusion were usually indicative of ciprofloxacin MICs of $\geqslant 12 \mathrm{mg} \mathrm{l}^{-1}$. Larger disc zones of $19 \mathrm{~mm}$ were associated with lower-level ciprofloxacin resistance, but these were less reliable indicators of resistance, as zones of a similar size $(20 \mathrm{~mm})$ were observed for two isolates that were sensitive according to MIC by Etest. Whilst our results indicated a cut-off zone size of $\leqslant 15 \mathrm{~mm}$ for resistance by the $1 \mu \mathrm{g}$ ciprofloxacin disc diffusion test using an inoculum of MacFarland standard 4, it is important that isolates that display intermediate zone sizes of $>15$ to $\leqslant 20 \mathrm{~mm}$ should be tested by an alternative method, such as Etest, to confirm full susceptibility. Whilst Etest and agar dilution are the preferred method for susceptibility testing of $H$. pylori, disc diffusion nevertheless offers an economical alternative to Etest that can be applied as a screening method in laboratories with insufficient throughput to justify agar dilution. 

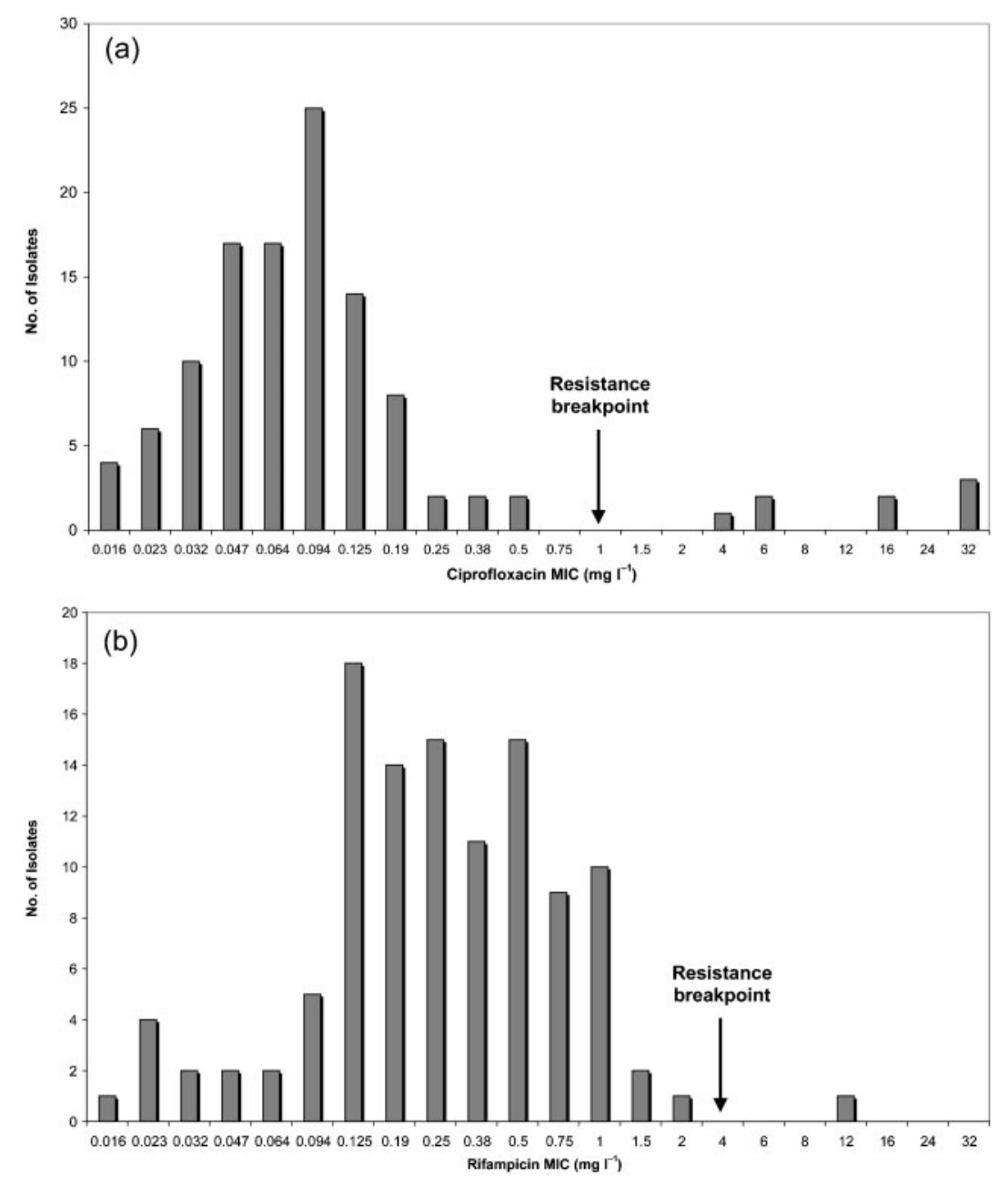

Fig. 1. MICs determined for 115 isolates of $H$. pylori for (a) ciprofloxacin and (b) rifampicin.

\section{Frequency of resistance to ciprofloxacin}

Of the 255 isolates tested by Etest and/or disc diffusion, $7.5 \%(n=19)$ were ciprofloxacin resistant. This study is the first to have surveyed ciprofloxacin susceptibility of isolates of $H$. pylori from adult patients in the UK, and our results showed that the overall resistance based on the data from patients with reported failures by standard eradication regimens was $7.5 \%$, even when isolates were resistant to both metronidazole and clarithromycin (dually resistant). In the treatment failure patients, dual resistance was a feature of $88 \%(106 / 121)$ of isolates, whereas we reported previously that dual resistance was less than $10 \%$ for isolates from UK patients that were considered unlikely to have received previous eradication therapy based on the available clinical information (Chisholm \& Owen, 2008). Furthermore, ciprofloxacin resistance was not a feature of the 10 exclusively metronidazole-resistant isolates and the two exclusively clarithromycin-resistant isolates from the treatment failure patients. Overall, the findings indicated that failed $H$. pylori eradication by standard treatment regimens had not selected specifically for ciprofloxacin resistance.
Our $H$. pylori resistance rate for ciprofloxacin is consistent with fluoroquinolone resistance rates of less than $10 \%$ in various countries including Germany, where a $9 \%$ ciprofloxacin resistance rate was reported in isolates from patients who had failed eradication therapy (Heep et al., 2000a). Furthermore, a subsequent analysis provided evidence of a temporal trend in ciprofloxacin resistance, with rates for isolates from other German treatment failure patients rising from $11 \%$ in 2003 to $22 \%$ in 2005 (Glocker et al., 2007b). The interpretation of direct comparisons between datasets needs to take into account differences in the types of patient populations studied and local treatment practices. For example, studies of ciprofloxacin resistance rates in Bulgaria (Boyanova et al., 2008), Japan (Fujimura et al., 2004), Egypt (Sherif et al., 2004) and Iran (Falsafi et al., 2004) included analyses of pre-treatment populations, and some included paediatric as well as adult patients. Interestingly, other independent studies have highlighted markedly different resistance rates within a particular country - for instance in France rates of $3 \%$ (Tankovic et al., 2003) and $17 \%$ (Cattoir et al., 2007), and likewise in Japan rates of $5.5 \%$ (Fujimura et al., 2004) and $15 \%$ (Miyachi et al., 2006). 
Table 1. Characteristics of gyrA ORDR mutations in isolates of $H$. pylori with a ciprofloxacin (CIP)-resistant phenotype

\begin{tabular}{|c|c|c|c|c|}
\hline gyrA mutation & CIP MIC (mg l-1) & $1 \mu \mathrm{g}$ disc zone size $(\mathrm{mm})$ & Isolate no. & Biopsy \\
\hline Asp91Gly & 2 & 19 & H4281 & Post-TF \\
\hline Asp91Gly & 4 & 19 & H4573 & Post-TF \\
\hline Asp91Gly & 12 & 14 & H061240430 & $\mathrm{NK}$ \\
\hline Asp91Gly & 16 & 0 & H051660640 & Post-TF \\
\hline Asp91Gly & 32 & 0 & H053880517 & Post-TF \\
\hline Asp91Gly & $>32$ & 0 & H042580533 & Post-TF \\
\hline Asp91Asn & 16 & 0 & H041960697 & Post-TF \\
\hline Asp91Asn & 16 & 11 & H064400334 & NK \\
\hline Asp91Asn & 32 & 0 & H060420429 & NK \\
\hline Asp91Asn & $>32$ & 0 & H062040554 & NK \\
\hline Asp91Tyr & 32 & 12 & H064160518 & NK \\
\hline Asp91Tyr & $>32$ & 0 & H064040339 & NK \\
\hline Asn87Lys & 6 & 9 & H050780467 & Post-TF \\
\hline Asn87Lys & 32 & 0 & H064640499 & Post-TF \\
\hline Asn87Lys & $>32$ & 0 & H053140361 & Post-TF \\
\hline Asn87Lys & $>32$ & 0 & H064160517 & $\mathrm{NK}$ \\
\hline Ala88Val & $>32$ & 0 & H062160313 & Post-TF \\
\hline None & $>32$ & 0 & H045160162 & NK \\
\hline
\end{tabular}

${ }^{\star}$ Clinical details provided indicated that gastric biopsy was taken post-treatment failure (Post-TF). Details for other cases were not known (NK). Each isolate was from an unrelated case.

It is unclear until more data are available whether these differences indicate a true temporal trend or population selection effects. Significantly higher levels of fluoroquinolone resistance have been reported in some countries, with rates as high as $20 \%$ in Iran (Falsafi et al., 2004) and $33 \%$ in Korea (Kim et al., 2004). The reason for these higher rates is unclear, but they may reflect different patterns of fluoroquinolone usage in those countries. For instance ciprofloxacin use in outpatients is lower in the UK relative to many other European countries (Muller et al., 2007).

Rescue treatment regimens based on levofloxacin have been used widely as an alternative to ciprofloxacin, as levofloxacin is absorbed in tissues more efficiently and has fewer side effects. There is evidence that ciprofloxacin-resistant strains of $H$. pylori exhibit cross-resistance to levofloxacin (Bogaerts et al., 2006). Although not investigated in the present study, previous investigations of levofloxacin resistance have reported rates of $8.8 \%$ in the USA (Carothers et al., 2007), $11.5 \%$ in China (Lee et al., 2008), $15 \%$ in Japan (Miyachi et al., 2006) and $19 \%$ in Italy (Zullo et al., 2007). In Italy, a levofloxacin resistance rate of $22 \%$ was demonstrated in 67 isolates with resistance to both clarithromycin and metronidazole (Branca et al., 2004). Carothers et al. (2007) found that levofloxacin resistance in the USA was associated with prior fluoroquinolone usage over the previous 10 years and with the total number of courses.

\section{Characterization of gyrA mutations in ciprofloxacin-sensitive and -resistant isolates}

The QRDR of gyrA was sequenced in 50 isolates, 18 of which were ciprofloxacin resistant, whilst the remaining 32 were ciprofloxacin sensitive. With one exception (strain H045160162), all of the ciprofloxacin-resistant isolates contained mutations in the QRDR of gyrA (Table 1) resulting in amino acid substitutions. Most (12/18, 67\%) isolates with a resistant phenotype contained mutations at aa 91, where Asp mutated to Gly $(n=6)$, to Asn $(n=4)$ or to Tyr $(n=2)$. The gyrA QRDR of the remaining seven isolates contained single substitutions of Asn87 $\rightarrow$ Lys $(n=4) \quad$ or Ala88 $\rightarrow$ Val $(n=1)$. The gyrA amino acid sequence was wild-type for the majority $(91 \%)$ of ciprofloxacin-sensitive isolates, and matched the QRDR sequence in GenBank (accession no. L29481; Moore et al., 1995). Not all mutations in the gyrA QRDR were associated with resistance, as three isolates with a ciprofloxacinsensitive phenotype (MICs ranging from 0.016 to $0.19 \mathrm{mg}$ $\left.1^{-1}\right)$ contained the Asn87 $\rightarrow$ Thr substitution.

Molecular markers for fluoroquinolone resistance in $H$. pylori are well documented (Cattoir et al., 2007; Glocker et al., 2007b) and we have confirmed that all but one of the UK isolates with a ciprofloxacin-resistant phenotype contained mutations in the QRDR of the gyrA gene. The particular mutations observed have all been reported previously (Moore et al., 1995; Bogaerts et al., 2006; Glocker et al., 2007b) and were consistent with the most common mutations being located at aa 91, as found in isolates from France (Tankovic et al., 2003; Cattoir et al., 2007) and Japan (Fujimura et al., 2004). Substitutions at aa 87 also were observed but not at the higher frequency noted in two large surveys of ciprofloxacin resistance in Belgium (Bogaerts et al., 2006) and Germany (Glocker \& Kist, 2004), suggesting geographical variation in gyrA 
QRDR genotype distribution. The significance of such variations is unclear, but it has been reported that certain mutations might be linked to a higher level of resistance. For instance, analysis of Belgian isolates indicated that two specific mutations (Asn87 $\rightarrow$ Ile and Asp91 $\rightarrow$ Asn) were consistently associated with higher MICs $\left(>32 \mathrm{mg} \mathrm{l}^{-1}\right)$ (Bogaerts et al., 2006). However, we were unable to correlate the mutation type of any UK isolate with MIC (Etest), and only one of the four isolates containing the gyrA Asp91 $\rightarrow$ Asn marker also exhibited a similarly high MIC. Knowledge of these common mutations is important as it provides the basis for designing real-time PCR assays that can be used to provide a rapid susceptibility result for culture (Glocker \& Kist, 2004) and offers the potential for direct application to gastric biopsies that have proved culture-negative - an approach already used successfully for clarithromycin and tetracycline testing (Chisholm \& Owen, 2008).

\section{Identification of rifampicin-resistant isolates of H. pylori}

Rifampicin MICs were more difficult to determine as faint fading edges of growth on blood agar medium were observed for cultures of most of the 115 isolates tested. A range of MICs was observed for sensitive isolates $\left(0.016-2 \mathrm{mg} \mathrm{l}^{-1}\right)$ (Fig. 1b). The $\mathrm{MIC}_{90}$ and $\mathrm{MIC}_{50}$ values of the isolates examined were 1.0 and $0.25 \mathrm{mg} \mathrm{l}^{-1}$, respectively (median $0.25 \mathrm{mg} \mathrm{l}^{-1}$, mode $0.125 \mathrm{mg} \mathrm{l}^{-1}$ ). One isolate (H062600603) was initially resistant (MIC $6 \mathrm{mg} \mathrm{l}^{-1}$, zone of inhibition of $0 \mathrm{~mm}$ ) but this was not stable on repeat testing (MIC $1 \mathrm{mg}$ $\mathrm{1}^{-1}$ ). Another isolate (H4573) was the only one in this set that was resistant by Etest (MIC $12 \mathrm{mg} \mathrm{l}^{-1}$ ). Disc diffusion zones of inhibition were again difficult to demarcate due to the faint fading edges of growth, and ranged from 18 to $49 \mathrm{~mm}$ (mean $31 \mathrm{~mm}$, mode $30 \mathrm{~mm}$ ) for sensitive isolates $(n=31)$. The range included the isolate considered resistant by Etest that gave an inhibition zone of $26 \mathrm{~mm}$.

\section{Frequency of resistance to rifampicin}

$H$. pylori isolates were tested for susceptibility to rifampicin, as rifamycin derivative-based therapies have been shown to provide a highly effective and well-tolerated alternative eradication strategy (Gisbert \& Pajares, 2005; Gisbert et al., 2003; Toracchio et al., 2005). Although there are no reports on the use of rifamycin-based therapies in the UK, our findings show that $H$. pylori resistance to this drug was $<1 \%$, even when isolates from treatment failures by conventional therapies were included. Only two isolates $(2 / 255,0.8 \%)$ displayed possible resistance to rifampicin in the Etest and/or disc diffusion assays used, so we concluded that prevalence of resistance to this agent is currently very low in the UK. There is a general paucity of information on rifampicin/rifabutin resistance of isolates from other European countries, but a recent study in Germany reported an overall resistance rate of $1.4 \%$ and noted that the majority of resistant strains were isolated from patients after treatment failure (Glocker et al., 2007a).
The technical aspects of testing the susceptibility of $H$. pylori to rifampicin have not hitherto been critically assessed, as inclusion of the drug in $H$. pylori eradication regimens is a relatively recent development. We observed that the precise MICs of the two rifampicin-resistant isolates were difficult to define, as cultures exhibited a faint fading edge of growth on agar plates. Repeat MIC testing of the isolates failed to resolve the problem encountered with interpretation, particularly as the disc diffusion test indicated resistance in only one of these isolates. As rifampicin susceptibility testing by Etest has been described to date in only two studies (Fujimura et al., 2002; Heep et al., 2002), it is important to develop standardized laboratory test methods and interpretative criteria. The possibility that difficulties in determining susceptibility in the current study could be overcome by alternative methods using different media or inocula cannot be excluded. Other less widely used methods of susceptibility testing such as agar dilution (Wang et al., 2001) provide an alternative means of identifying resistant isolates and of validating the disc diffusion and Etest.

\section{Investigation of rpoB for mutations associated with rifampicin resistance}

The $r p o B$ sequences at the $5^{\prime}$ end of $r p o B$ and in the $r p o B$ cluster I and II regions of four isolates of $H$. pylori that were either resistant (two isolates with MICs of 6 and $12 \mathrm{mg} \mathrm{l}^{-1}$ ) or potentially had reduced rifampicin susceptibility (two isolates with MICs of 1.5 and $2 \mathrm{mg}^{-1}$ but disc zone sizes of 12 and $13 \mathrm{~mm}$ ) and five randomly selected rifampicinsusceptible isolates (disc zone sizes of 22-28 mm) were examined. All of the sequences had the wild-type amino acid sequence and no substitutions were present at any of the amino acid positions in the regions previously reported to be associated with rifampicin/rifabutin resistance in $H$. pylori (Heep et al., 2000b, 2002; Wang et al., 2001; Glocker et al., 2007a). The use of molecular tests merits further investigation, as mutations in $H$. pylori rpoB at codons 525-545 and 586 have been shown to arise as a result of resistance induction (Heep et al., 1999; Wang et al., 2001). However, observations based on a single rifampicinresistant clinical isolate from Germany identified only one mutation $(\mathrm{V} 149 \mathrm{~F})$ at the $5^{\prime}$ end of $r p o B$ that was able to transform susceptible isolates to the resistant phenotype (Heep et al., 2000b). None of these documented mutations in $r p o B$ were found in either of the isolates with reduced susceptibility to rifampicin described in the current study, suggesting that the complete $r p o B$ genes of a larger number of isolates with resistant phenotypes need to be sequenced and that the potential contribution of other mechanisms to this resistance should be explored.

\section{Conclusions}

As the study included isolates of $H$. pylori from patients who had failed eradication attempts by standard regimens, we conclude that such 'problem' patients could potentially 
benefit from inclusion of ciprofloxacin/levofloxacin or rifampicin in future eradication therapy, as resistance rates are low in the UK. The isolates investigated were from gastric biopsy samples referred for routine testing and were not part of a systematic surveillance study; consequently, it is not possible to draw any conclusions about the general situation on $H$. pylori resistance to these antibiotics in the UK. However, future studies need to aim to include isolates from a more representative selection of cases and to ensure that resistance data on levofloxacin are available.

\section{REFERENCES}

Bogaerts, P., Berhin, C., Nizet, H. \& Glupczynski, Y. (2006). Prevalence and mechanisms of resistance to fluoroquinolones in Helicobacter pylori strains from patients living in Belgium. Helicobacter 11, 441-445.

Boyanova, L., Gergova, G., Knikolov, R., Davidkov, L., Kamburov, V., Jelev, C. \& Mitov, I. (2008). Prevalence and evolution of Helicobacter pylori resistance to 6 antimicrobial agents over 12 years and correlation between susceptibility testing methods. Diagn Microbiol Infect Dis 60, 409-415.

Branca, G., Spanu, T., Cammarota, G., Schito, A. M., Gasparini, A., Gasparini, G. B. \& Fadda, G. (2004). High levels of dual resistance to clarithromycin and metronidazole and in vitro activity of levofloxacin against Helicobacter pylori isolates from patients after failure of therapy. Int J Antimicrob Agents 24, 433-438.

Cabrita, J., Oleastro, M., Matos, R., Manhente, A., Cabral, J., Barros, R., Lopes, A. I., Ramalho, P., Neves, B. C. \& Gurreiro, A. S. (2000). Features and trends in Helicobacter pylori antibiotic resistance in Lisbon area, Portugal (1990-1999). J Antimicrob Chemother 46, 1029-1031.

Carothers, J. J., Bruce, M. G., Hennessy, T. W., Bensler, M., Morris, J. M., Reasonover, A. L., Hurlburt, D. A., Parkinson, A. M., Coleman, J. M. \& McMahon, B. J. (2007). The relationship between previous fluoroquinone use and levofloxacin resistance in Helicobacter pylori infection. Clin Infect Dis 44, e5-e8.

Cattoir, V., Nectoux, J., Lascols, C., Deforges, L., Delchier, J. C., Megraud, F., Soussy, C. J. \& Cambau, E. (2007). Update on fluoroquinolone resistance in Helicobacter pylori: new mutations leading to resistance and first description of a $g y r A$ polymorphism associated with hypersusceptibility. Int J Antimicrob Agents 29, 389396.

Chisholm, S. A. \& Owen, R. J. (2008). Application of polymerase chain reaction based assays for rapid identification and antibiotic resistance screening of Helicobacter pylori in gastric biopsies. Diagn Microbiol Infect Dis 61, 67-71.

Chisholm, S. A., Teare, E. L., Davies, K. \& Owen, R. J. (2007) Surveillance of primary antibiotic resistance of Helicobacter pylori at centres in England (mid-Essex) and Wales (Gwynedd) over a six-year period (2000-2005). Euro Surveill 12, E3-E4.

Dore, M. P., Leandro, G., Realdi, G., Sepulveda, A. R. \& Graham, D. Y. (2000). Effect of pretreatment antibiotic resistance to metronidazole and clarithromycin on outcome of Helicobacter pylori therapy: a meta-analytical approach. Dig Dis Sci 45, 68-76.

Elviss, N. C., Owen, R. J., Xerry, J., Walker, A. M. \& Davies, K. (2004). Helicobacter pylori resistance patterns and genotypes in adult dyspeptic patients from a regional population in North Wales. J Antimicrob Chemother 54, 435-440.

Elviss, N. C., Owen, R. J., Breathnach, A., Palmer, C. \& Shetty, N. (2005). Helicobacter pylori antibiotic-resistance patterns and risk factors in adult dyspeptic patients from ethnically diverse populations in central and south London during 2000. J Med Microbiol 54, 567574.

Falsafi, T., Mobasheri, F., Nariman, F. \& Najafi, M. (2004). Susceptibilities to different antibiotics of Helicobacter pylori strains isolated from patients at the pediatric medical center of Tehran, Iran. J Clin Microbiol 42, 387-389.

Fujimura, S., Kato, S., Kawamura, T. \& Watanabe, A. (2002). In vitro activity of rifampicin against Helicobacter pylori isolated from children and adults. J Antimicrob Chemother 49, 541-543.

Fujimura, S., Kato, S., linuma, K. \& Watanabe, A. (2004). In vitro activity of fluoroquinolone and the gyrA gene mutation in Helicobacter pylori strains isolated from children. I Med Microbiol 53, 1019-1022.

Gisbert, J. P. \& Pajares, J. M. (2005). Helicobacter pylori "rescue" therapy after failure of two eradication treatments. Helicobacter 10, 363-372.

Gisbert, J. P., Calvet, X., Bujanda, L., Marcos, S., Gisbert, J. L. \& Pajares, J. M. (2003). 'Rescue' therapy with rifabutin after multiple Helicobacter pylori treatment failures. Helicobacter 8, 90-94.

Glocker, E. \& Kist, M. (2004). Rapid detection of point mutations in the gyrA gene of Helicobacter pylori conferring resistance to ciprofloxacin by a fluorescence resonance energy transfer-based real-time PCR approach. J Clin Microbiol 42, 2241-2246.

Glocker, E., Bogdan, C. \& Kist, M. (2007a). Characterization of rifampicin-resistant clinical Helicobacter pylori isolates from Germany. J Antimicrob Chemother 59, 874-879.

Glocker, E., Stueger, H. P. \& Kist, M. (2007b). Quinolone resistance in Helicobacter pylori isolates in Germany. Antimicrob Agents Chemother 51, 346-349.

Go, M. F. (2002). Review article: natural history and epidemiology of Helicobacter pylori infection. Aliment Pharmacol Ther 16 (Suppl. 1), 3-15.

Heep, M., Beck, D., Bayerdorffer, E. \& Lehn, N. (1999). Rifampin and rifabutin resistance mechanism in Helicobacter pylori. Antimicrob Agents Chemother 43, 1497-1499.

Heep, M., Kist, M., Strobel, S., Beck, D. \& Lehn, N. (2000a). Secondary resistance among 554 isolates of Helicobacter pylori after failure of therapy. Eur J Clin Microbiol Infect Dis 19, 538-541.

Heep, M., Rieger, U., Beck, D. \& Lehn, N. (2000b). Mutations in the beginning of the $r p o B$ gene can induce resistance to rifamycins in both Helicobacter pylori and Mycobacterium tuberculosis. Antimicrob Agents Chemother 44, 1075-1077.

Heep, M., Lehn, N., Brandstatter, B., Rieger, U., Senzenberger, S. \& Wehrl, W. (2002). Detection of rifabutin resistance and association of $r p o B$ mutations with resistance to four rifamycin derivatives in Helicobacter pylori. Eur J Clin Microbiol Infect Dis 21, 143-145.

Jenks, P. J. (2002). Causes of failure of eradication of Helicobacter pylori. BMJ 325, 3-4.

Kim, J. M., Kim, J. S., Jung, H. C., Kim, N., Kim, Y. J. \& Song, I. S. (2004). Distribution of antibiotic MICs for Helicobacter pylori strains over a 16-year period in patients from Seoul, South Korea. Antimicrob Agents Chemother 48, 4843-4847.

Kumala, W. \& Rani, A. (2006). Patterns of Helicobacter pylori isolate resistance to fluoroquinolones, amoxicillin, clarithromycin and metronidazole. Southeast Asian J Trop Med Public Health 37, 970-974.

Lee, C. C., Lee, V. W., Chan, F. K. \& Ling, T. K. (2008). Levofloxacinresistant Helicobacter pylori in Hong Kong. Chemotherapy 54, 50-53.

Malfertheiner, P., Megraud, F., O'Morain, C., Bazzoli, F., El-Omar, E., Graham, D., Hunt, R., Rokkas, T., Vakil, N. \& Kuipers, E. J. (2007). 
Current concepts in the management of Helicobacter pylori infection: The Maastricht III Consensus Report. Gut 56, 722-781.

Miyachi, H., Miki, I., Aoyama, N., Shirasaka, D., Matsumoto, Y., Toyoda, M., Mitani, T., Morita, Y., Tamura, T. \& other authors (2006). Primary levofloxacin resistance and gyrA/B mutations among Helicobacter pylori in Japan. Helicobacter 11, 243-249.

Moore, R. A., Beckthold, B., Wong, S., Kureishi, A. \& Bryan, L. E. (1995). Nucleotide sequence of the gyrA gene and characterization of ciprofloxacin-resistant mutants of Helicobacter pylori. Antimicrob Agents Chemother 39, 107-111.

Muller, A., Coenen, S., Monnet, D. L., Goossens, H. \& ESAC Project Group (2007). European Surveillance of Antimicrobial Consumption (ESAC): outpatient antibiotic use in Europe, 1998-2005. Euro Surveill 12, E071011.1.

Reece, R. J. \& Maxwell, A. (1991). DNA gyrase: structure and function. Crit Rev Biochem Mol Biol 26, 335-375.

Sherif, M., Mohran, Z., Fathy, H., Rockabrand, D. M., Rozmajzl, P. J. \& Frenck, R. W. (2004). Universal high-level primary metronidazole resistance in Helicobacter pylori isolated from children in Egypt. J Clin Microbiol 42, 4832-4834.

Suerbaum, S. \& Michetti, P. (2002). Helicobacter pylori infection. $N$ Engl J Med 347, 1175-1186.

Tankovic, J., Lascols, C., Sculo, Q., Petit, J.-C. \& Soussy, C.-J. (2003). Single and double mutations in $g y r A$ but not in $g y r B$ are associated with low- and high-level fluoroquinolone resistance in Helicobacter pylori. Antimicrob Agents Chemother 47, 3942-3944.

Toracchio, S., Capodicasa, S., Soraja, D. B., Cellini, L. \& Marzio, L. (2005). Rifabutin based triple therapy for eradication of $H$. pylori primary and secondary resistant to tinidazole and clarithromycin. Dig Liver Dis 37, 33-38.

Wang, G., Wilson, T. J., Jiang, Q. \& Taylor, D. E. (2001). Spontaneous mutations that confer antibiotic resistance in Helicobacter pylori. Antimicrob Agents Chemother 45, 727-733.

Zullo, A., Perna, F., Hassan, C., Ricci, C., Saracino, I., Morini, S. \& Vaira, D. (2007). Primary antibiotic resistance in Helicobacter pylori strains isolated in northern and central Italy. Aliment Pharmacol Ther 25, 1429-1434. 